\title{
Emission Imaging of Pulsed Plasma Plumes Emerging Through a Transversal Hole in the Hollow Cathode of a DC Reactor
}

\author{
Isabel Tanarro
}

\begin{abstract}
A sequence of images shows the emission of brief pulsed plasma plumes emerging laterally from a small hole in the wall of a dc hollow-cathode reactor. The orifice diameter is comparable to the plasma sheath thickness. The images highlight the transient behavior under particular conditions of these plasma events. They also extend current predictions for RF discharges on the distributions of ions flowing through small apertures to the case of hollow-cathode de discharges.
\end{abstract}

Index Terms-Optical imaging, plasma molding, plasma plumes, plasma stability.

$\mathbf{T}$ HE CONTROLLED extraction of active species from low-pressure plasmas through small apertures finds application in different fields like ion beam source generation, ion etching, ion implantation, afterglows, and so on. In the diagnostic of plasmas by differentially pumped mass spectrometric techniques, ions and neutrals must be sampled through a small orifice to minimize perturbations to the plasma. Plasma molding around surfaces is relevant for the coating and surface conditioning of objects with irregular shapes. Moreover, scaling these phenomena to higher pressures and smaller size holes is, at present, a very active subject in the growing field of microdischarges.

Angular and energy distributions of ion beams extracted from RF plasmas through holes of different sizes have been modeled recently by means of a particle-in-cell simulation [1] and have been experimentally studied in RF high-density plasmas [2]. According to these studies, the angular distribution of the extracted beam depends on the orifice diameter $d$, so that the width of this distribution increases with the ratio of hole diameter to sheath thickness $s$. When $d \ll s$, the plasma-sheath interface in the neighborhood of the orifice is essentially planar. In the case of $d \gg s$, the plasma "leaks" through the orifice, giving rise to a fairly isotropic plasma plume diverging from the surface normal. In the intermediate case, the plasma-sheath

Manuscript received November 8, 2007; revised January 28, 2008. This work was supported by the Ministry of Education of Spain under Grants ENE200614577, FIS2004-00456, and FIS2007-61686.

The author is with the Instituto de Estructura de la Materia, Consejo Superior de Investigaciones Científicas, 28006 Madrid, Spain (e-mail: itanarro@iem.cfmac.csic.es).

Color versions of one or more of the figures in this paper are available online at http://ieeexplore.iee.org.

Digital Object Identifier 10.1109/TPS.2008.922484 meniscus "bends" over the hole, and the plasma is expected to start bulging into the aperture. Similar relationships between characteristic dimensions and discharge shape are found in hollow-cathode dc discharges and are, at present, a subject of interest for the development of microplasmas [3].

In this paper, the effect of a small hole in the wall of a large hollow-cathode dc reactor with an orifice diameter that is just comparable to the plasma sheath thickness is illustrated. The most remarkable observation is the unstable behavior of the plasma plumes which, under certain pressures and electric currents, emerge through the orifice in a pulsed form.

The reactor consisted of a stainless steel cylinder (diameter $=10 \mathrm{~cm}$ and length $=20 \mathrm{~cm}$ ) which acted as cathode, with a central rod acting as anode. This arrangement was placed inside a larger cylindrical vacuum vessel with a frontal glass window for observation. The lateral orifice in the cathode wall had a diameter $d=6 \mathrm{~mm}$. The open basis of the cathode in front of the observation window was covered with a metallic mesh of wires forming square apertures of $2 \times 2 \mathrm{~mm}^{2}$. The vacuum vessel and the cathode were electrically grounded, and the dc discharge was sustained in a small flux of natural air at a pressure of $0.08 \mathrm{mbar}$, a current of $100 \mathrm{~mA}$, and a voltage of $400 \mathrm{~V}$. Under these conditions, the negative glow had a very uniform, diffuse, and stable appearance and was wholly confined inside the cathode, except for the aforementioned plasma plumes, which emerged suddenly through the hole and disappeared, without appreciable current and voltage disturbances $(\leq 2 \%)$. A sequence of images of this behavior is shown in Fig. 1. The photographs have been extracted from a film obtained with a digital camera (Canon Power Shot A60), with an acquisition frequency of 15 frames/s. Individual plume events lasted for no more than one or two consecutive frames, i.e., $\sim 0.1 \mathrm{~s}$, with irregular time intervals among them of $\sim 0.1-1 \mathrm{~s}$, and developed up to a very repetitive maximum length of $\sim 2 \mathrm{~cm}$. A decrease in the discharge current, which is associated with a decrease in electron density and a growth of the plasma sheath thickness, led to the disappearance of the plasma plume. On the contrary, a slow current increase of up to $\sim 150 \mathrm{~mA}$ caused the plume to appear more frequently and, finally, to be continuous.

A detailed explanation of the observed behavior is out of the scope and length of this paper; however, a comparison of the orifice diameter with the plasma sheath thickness for the conditions of Fig. 1 strongly suggests that the plume appearance and its transient behavior are due to the fact that, 

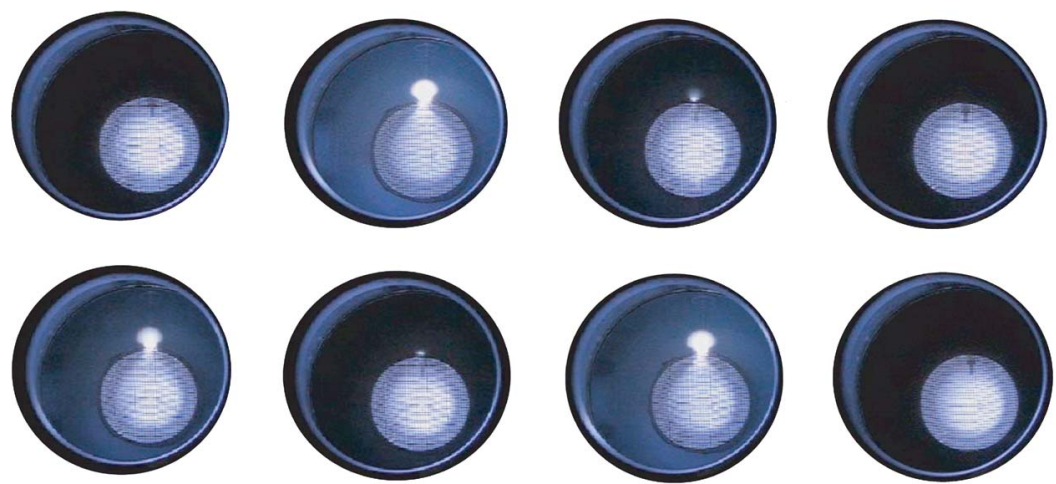

Fig. 1. Brief plasma plumes emerging suddenly from a hole made in the wall of a hollow-cathode reactor toward a larger vacuum vessel. The diameter of the hole is comparable to the plasma sheath thickness.

when $d$ and $s$ are comparable, the system is in an intermediate unstable region, in which very small experimental fluctuations can lead to oscillations between two possible and opposite behaviors.

The plasma sheath thickness $s$ depends on the Debye length $\lambda_{D}$ and the electric potential $V$ and is given [4] by

$$
s=(\sqrt{2} / 3) \lambda_{D}\left(2 V / T_{e}\right)^{0.75} \text {. }
$$

Radial distributions of charge density $N_{e}$ and electron energy $T_{e}$ were measured previously with a double Langmuir probe in a large hollow-cathode reactor that is very similar to the present one but without the external vacuum vessel [5]. For the conditions of Fig. $1(P=0.08 \mathrm{mbar}$ and $I=100 \mathrm{~mA})$, the electron temperature was $T_{e}=3 \mathrm{eV} . N_{e}$ showed a maximum value of $1.5 \cdot 10^{10} \mathrm{~cm}^{-3}$ at the axis of the reactor and decreased in the vicinity of the walls to a value of $\sim 5 \cdot 10^{9} \mathrm{~cm}^{-3}$. The corresponding Debye length near the cathode wall was then $\lambda_{D} \sim 0.2 \mathrm{~mm}$, and the sheath thickness $s$ was $\sim 6 \mathrm{~mm}$, i.e., it was comparable to the diameter of the hole. Further experiments with single lateral orifices of smaller diameters led to consistent results: No emerging plumes were observed under the present $(P, I)$ conditions. On the other hand, the square orifices of the metallic mesh installed in front of the cathode, with a size that was smaller than the sheath thickness, confined completely the plasma. Therefore, the results shown in Fig. 1 confirm the dependence of the plume formation on the $d / s$ ratio predicted in [1] and [2].
In conclusion, in this paper, the effect on the plasma of a hole made in the wall of a hollow-cathode reactor with a diameter that is comparable to the plasma sheath thickness has been shown. Most of the time, the plasma is not perturbed by the hole, as expected for an aperture with smaller diameter. Nevertheless, during short but significant time intervals, transient plasma plumes, divergent and quite isotropic, emerge through the aperture, as predicted for a hole with a diameter that is larger than the plasma sheath thickness.

\section{ACKNOWLEDGMENT}

The author would like to thank V. J. Herrero for carefully reading this paper and $\mathrm{M}$. A. Moreno for his help in editing the figure.

\section{REFERENCES}

[1] S. K. Nam, V. M. Donnelly, and D. J. Economou, "Particle-in-cell simulation of ion flow through a hole in contact with plasma," IEEE Trans. Plasma Sci., vol. 33, no. 2, pp. 232-233, Apr. 2005.

[2] C. K. Kim and D. J. Economou, "Plasma molding over surface topography: Energy and angular distribution of ions extracted out of large holes," J. Appl. Phys., vol. 91, no. 5, pp. 2594-2603, 2002.

[3] K. H. Becker, K. H. Schoenbach, and J. G. Eden, "Microplasmas and applications," J. Phys. D, Appl. Phys., vol. 39, no. 3, pp. R55-R70, Feb. 2006.

[4] M. A. Lieberman and A. J. Lichtenberg, Principles of Plasma Discharges and Materials Processing. New York: Wiley, 1994.

[5] M. Castillo, I. Méndez, A. M. Islyaikin, V. J. Herrero, and I. Tanarro, "Low pressure DC air plasmas. Investigation of neutral and ion chemistry," J. Phys. Chem. A, vol. 109, no. 28, pp. 6255-6263, 2005. 